\title{
Laboreal
}

Volume $13 \mathrm{~N}^{\circ} 1$ | 2017

Varia

\section{Organización del trabajo y sufrimiento psíquico en las actividades de servicio: el caso de los call centers en Argentina}

Organização do trabalho e sofrimento psíquico nas atividades de serviço: o caso dos call centers na Argentina

Organisation du travail et souffrance psychique dans les activités de service : le cas des centres d'appels en Argentine

Work organisation and psychic suffering in service activities : the case of Argentine call centres

\section{Patricio Nusshold}

\section{(2) OpenEdition}

\section{Journals}

\section{Edición electrónica}

URL: http://journals.openedition.org/laboreal/1981

DOI: 10.4000/laboreal. 1981

ISSN: 1646-5237

\section{Editor}

Universidade do Porto

\section{Referencia electrónica}

Patricio Nusshold, « Organización del trabajo y sufrimiento psíquico en las actividades de servicio: el caso de los call centers en Argentina », Laboreal [En línea], Volume $13 N^{0} 1$ | 2017, Publicado el 01 julio 2017, consultado el 23 septiembre 2020. URL : http://journals.openedition.org/laboreal/1981 ; DOI : https://doi.org/10.4000/laboreal.1981

Este documento fue generado automáticamente el 23 septiembre 2020

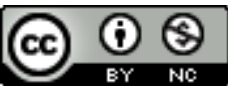

Laboreal está licenciado com uma Licença Creative Commons - Atribuição-NãoComercial 4.0 Internacional. 


\section{Organización del trabajo y sufrimiento psíquico en las actividades de servicio: el caso de los call centers en Argentina}

Organização do trabalho e sofrimento psíquico nas atividades de serviço: o caso dos call centers na Argentina

Organisation du travail et souffrance psychique dans les activités de service : le cas des centres d'appels en Argentine

Work organisation and psychic suffering in service activities : the case of Argentine call centres

\section{Patricio Nusshold}

\section{REFERENCIA}

Nusshold, P. (2015). Organisation du travail et souffrance psychique dans les activités de service. Le cas des centres d'appels en Argentine. Thèse de doctorat en psychologie du travail, préparée en cotutelle : Universidad de Buenos Aires et École doctorale Abbé Grégoire, Centre de recherche sur le travail et le développement, Conservatoire National des Arts et Métiers, Paris.

\section{NOTA DEL EDITOR}

http://dx.doi.org/10.15667/laborealxiii0117pnes Manuscrito recibido en: Agosto/2016 Aceptado tras peritaje: Mayo/2017 
Júri da tese: Francis Six (Presidente); Christophe Dejours e Mario Poy (Orientadores); Laerte Sznelwar e Eduardo Keegan (Arguentes).

\section{Introducción}

1 La tesis doctoral que se presenta a continuación analiza diversos modelos de intervención que buscan generar procesos de transformación en el mundo del trabajo y sus consecuencias para la salud de los trabajadores. El foco del estudio se ubica en particular en el impacto sobre la salud mental. Este trabajo nace a partir de interrogantes en torno a la práctica en el terreno en psicodinámica del trabajo (PDT) y en ergonomía. La mirada de los especialistas en la evaluación de los riesgos psicosociales (RPS), la de los ergónomos y la de los clínicos del trabajo son divergentes no sólo en su concepción del trabajo sino también de la salud, e incluso en lo que puede significar transformar el trabajo. Se presentan sucintamente los objetivos de esta investigación, la metodología elegida con sus ventajas y sus límites y, por último, los resultados obtenidos. A modo de conclusión, se busca profundizar la cuestión de la acción y las posibilidades de transformación de las situaciones de trabajo patógenas.

\section{Objetivos y metodología}

2 El trabajo de campo de la tesis, que se concentra en la actividad en call centers, sirvió como punto de partida para estudiar dos grandes ejes: (1.) las consecuencias del trabajo en los call centers para la salud mental y (2.) los diferentes enfoques de esta problemática con miras a su transformación.

3 Entonces, los objetivos de esta tesis son:

- investigar sobre las diferentes formas de cooperación entre los operadores de los call centers,

- identificar las estrategias de defensa desplegadas por los trabajadores a partir de la brecha entre la tarea descripta y el trabajo real que deben enfrentar en su actividad profesional,

- y comparar la manera de estructurar la organización del trabajo y sus consecuencias sobre la salud de los trabajadores de los call centers en Argentina, con las de otros países de Latinoamérica y de Europa a partir de los resultados de nuestro trabajo de campo.

Se analizó la situación de los call centers en Argentina a partir de una metodología que tuvo ventajas y límites.

Hubo tres momentos diferenciados. En primer lugar, se realizaron visitas al terreno y un análisis global de la actividad mediante la metodología de la ergonomía en nueve plataformas telefónicas en Argentina, en Brasil y en Francia. Luego, se llevó a cabo un trabajo de campo más profundo en dos etapas:

- la primera, según una metodología cuantitativa, según el modelo de la evaluación de los riesgos psicosociales en dos call centers,

- y la segunda, aplicando la metodología de la psicodinámica del trabajo, partiendo de la demanda de un colectivo de teleoperadores.

La elección de diferentes metodologías resultó útil para comprender los abordajes, sus alcances, sus diferencias y sus límites, y para pensar los diversos modos de interacción posibles entre ellas. No obstante, esta estrategia se convirtió también en una 
restricción. Para poder profundizar la mirada de una de estas disciplinas, hubiera sido sin duda más eficaz elegir un único abordaje.

\section{Principales resultados}

7 Se presentan a continuación los resultados obtenidos en cada etapa.

8 Durante los cinco años del estudio, se realizaron visitas a nueve call centers en Brasil, en Argentina y en Francia. Gracias a estas visitas y experiencias, se pudo investigar en detalle la organización del trabajo en los call centres. Esta actividad económica no cesó de crecer y desarrollarse en Argentina. Se emplea principalmente una población de jóvenes de 18 a 25 años, segmento de la población que sufre en la actualidad el más alto porcentaje de desocupación. Por medio de estos análisis globales de la actividad, se identifica un modo general de estructurar la organización del trabajo común a los diferentes call centers. La distribución del espacio, las tareas, las escuchas, los scripts y los objetivos de desempeño en Argentina, en Francia y en Brasil presentan características similares.

9 Tras este primer análisis general, los abordajes previamente mencionados intentan encontrar respuestas al primer eje de esta investigación, es decir: la influencia del trabajo sobre la salud mental de los operadores.

Por medio de cuestionarios cuantitativos de evaluación de los riesgos psicosociales, se observan que los RPS los que representan las más importantes fuentes de estrés para estos colectivos son: las exigencias cognitivas en el trabajo; la imposibilidad de controlar el tiempo, y de adaptar su trabajo; la falta de apoyo de los superiores jerárquicos; una cierta fragilidad del grupo; un bajo grado de integración y; por último, la vivencia de su trabajo como falto de sentido.

11 Por su parte, la investigación en PDT confirmó que, en Argentina, los operadores de los call centers experimentan síntomas similares a los de las neurosis de las telefonistas, junto con trastornos músculo-esqueléticos. Los teleoperadores no están exentos de casos de burnout, depresión e incluso se suicidio en el lugar de trabajo que fueron descriptos a lo largo de la investigación colectiva. La percepción de un trabajo "basura" es muy cercana a la concepción de "trabajo sucio", descripta en otras actividades laborales (Lhuilier, 2005; Molinier, 2009).

12 Además, el trabajo de campo ha permitido identificar en el trabajo de call centers en Argentina la presencia de sufrimiento ético bajo diversas formas (Dejours, 1998/2006). La disposición a distorsionar la información y mentir para adaptarse parecen constituir características indispensables para llevar a cabo el trabajo. Las trabajadoras y los trabajadores de call centers de Argentina desarrollan estrategias individuales y colectivas de defensa para asegurar su servicio y no enfermarse.

13 La investigación permitió identificar algunas estrategias de defensa similares a las ya reconocidas por otros investigadores en poblaciones de call centers en otros países. En particular, encontramos también en Argentina defensas colectivas del orden de la infantilización de los trabajadores, la guerra de clanes y la negación del mal causado a los usuarios, tales como describen Rolo (2013) en teleoperadores en Francia, o Sznelwar, Abrahão y Mascia (2006) en Brasil. Se comprueba además la existencia de estrategias de defensa que difieren de las descriptas anteriormente en los call centers. Brindar un servicio en otro idioma, tendría un efecto narcicisante para el/la trabajador/a, pues 
pondría en evidencia su capacidad para comunicarse en un idioma extranjero. A su vez, trabajar para empresas y usuarios extranjeros más que para compatriotas, facilitaría a los operadores la necesidad de tomar distancia para realizar tareas que reprueban moralmente. Queda como posible eje a desarrollar en investigaciones futuras la influencia de la relación histórica de dominación entre países latinoamericanos y países "desarrollados" en el trabajo mediatizado para empresas y usuarios extranjeros.

Otro elemento novedoso que emana del trabajo de campo es la presencia de mecanismos de defensa que toman la forma de una reducción en el compromiso con del trabajo. A partir de la investigación colectiva en PDT, se pudo identificar una forma de desobediencia a la autoridad en los teleoperadores, que puede ir desde transgresiones menores hasta tomar la forma de un verdadero trabajo a reglamento. Esta estrategia de desobediencia a la autoridad, que encuentra algunos puntos en común con la descripta por Ganem (2012) en trabajadores hoteleros en la isla de Guadalupe, aparece también en esta población. Ante la falta de reconocimiento, que toma múltiples formas que van desde la falta de transparencia en la paga a controles médicos abusivos en caso de enfermedad, ésta parecería constituirse en otra de las principales estrategias para soportar este trabajo con poco margen de maniobra.

Pasando ahora al segundo eje de esta tesis: ¿qué aportes hacen los diferentes abordajes para pasar a la acción a partir de los datos que cada uno proporciona? Se trata de una cuestión no sólo teórica, relativa a cómo se generan datos, sino también y sobre todo de una cuestión relativa a la práctica, para los diferentes profesionales: ¿qué datos facilitan una evolución favorable del trabajo?

Riesgo psicosocial y sufrimiento no significan lo mismo. Uno de los principales problemas que se plantea cuando se asimila el concepto de sufrimiento a la noción de estrés o de RPS es la negación del lugar que la subjetividad y el sujeto -en el sentido del psicoanálisis- ocupan para la psicopatología y la psicodinámica del trabajo. Pierde visibilidad, a su vez, el lugar esencial que ocupan las estrategias de defensa en la determinación de los comportamientos y actitudes de los trabajadores (Dejours, 1980/1990). El modelo de los RPS, basado principalmente en la teoría del estrés, y los modelos de Karasek (1979; Karasek et al., 1998) y Siegrist et al. (2004; Siegrist, Wege, Pühlhofer, \& Wahrendorf, 2008), intenta evaluar, por una parte, los desencadenantes de estrés $\mathrm{y}$, por otro, la vivencia de los trabajadores respecto a su insatisfacción en el trabajo. A partir del modelo de los RPS, si al momento de responder a los cuestionarios, un trabajador o un colectivo de trabajadores proporciona respuestas globalmente positivas respecto de su trabajo, se puede inferir que el trabajo no tiene impacto negativo. No obstante, como lo ha demostrado la PDT, las estrategias defensivas poseen un lugar central en la percepción que los sujetos tienen de su situación laboral: que un trabajador responda positivamente no implica necesariamente que así sea, pues, la negación, por ejemplo, como estrategia de defensa, puede influenciar su respuesta e impedirle identificar los riesgos. De hecho, si fuese consciente de ciertos riesgos, es posible que no pudiese continuar a trabajar.

17 Los cuestionarios dirigidos a la evaluación de los RPS pusieron en evidencia una contradicción entre una voluntad aparente de encontrar soluciones, y la falta de cuestionamiento de algunas de las causas estructurales de los problemas de salud mental en el trabajo. Frente a la toma de conciencia de la importancia del problema, de la relación entre la salud mental de los trabajadores y la evolución del management, parecería que uno de los únicos abordajes dispuestos a encuadrar y generar las 
condiciones para una verdadera deliberación sobre estas cuestiones es la psicodinámica del trabajo. La mayoría de las demás intervenciones propuestas hoy en día, incluso ciertos enfoques biomecánicos de la ergonomía, parecen verse cada vez más influenciadas por las formas de evaluación cuantitativas (ya sea de los TMS o de los RPS) que ponen el eje de sus análisis en datos cuantitativos y no permiten discutir la riqueza del trabajo real y las formas de sufrimiento patógeno de forma tal que se facilite la apertura de las vías hacia la transformación del trabajo.

\section{A modo de conclusión: cambiar el trabajo}

Un interrogante que estuvo en el origen de este trabajo fue planteado por Dejours en el Congreso de la ODAM [1], en Sao Paulo en marzo de 2008. Él se preguntaba cómo podía ser posible que a pesar del intenso trabajo que realizamos estas últimas décadas, mejorando indiscutiblemente las condiciones materiales del trabajo, nos encontráramos hoy con un gran aumento del grado de sufrimiento en el trabajo como nunca antes había sido tan alto. Se ignoraba en ese momento, pero no pronto iniciarían las olas de suicidios acaecidos en Francia, Japón, Taiwán, China y otros países en el mundo, sin precedente alguno [2].

19 ¿A qué alude la frase "cambiar el trabajo"? Esta pregunta no tiene las mismas respuestas para la ergonomía que para la psicodinámica del trabajo. Para los especialistas en la evaluación de los RPS es una cuestión aún más enigmática, pues las recomendaciones posteriores a la presentación de los resultados que se realizan desde este abordaje, suelen ser bastante vagas, incluso en el documento del colegio de expertos creado en Francia a pedido del Ministerio de Trabajo (Askenazy et al., 2011).

Hoy en día, la ergonomía, por lo menos en Argentina, tiende a cada vez más a medir cuantitativamente el trabajo y estandarizar baremos, que a entender el trabajo efectivo y subjetivo de cada uno de los colectivos, las consecuencias sobre la salud de los trabajadores y sus estrategias defensivas.

21 El análisis de la demanda juega, en ese sentido, un rol crucial e ineludible. Trabajar a partir de una demanda constituye un punto compartido por la ergonomía de la actividad y la PDT. En PDT, cuando se consigue implementar una investigación colectiva a partir de una demanda bien trabajada, la investigación colectiva se orienta a la acción desde el inicio de nuestro trabajo. Emergen entonces situaciones concretas que se encuentran al origen del sufrimiento de ese colectivo de trabajadores en particular. Situaciones que los resultados cuantitativos no muestran y que pueden no tener relación con variables o riesgos imaginados a priori por los cuestionarios.

Que sujetos y equipos acepten cuestionarse acerca de su trabajo no es evidente. Supone estar dispuestos a poner en peligro un equilibrio que tomó tiempo construir, aun cuando el resultado del trabajar sea para algunos hacer la experiencia de diversas formas de sufrimiento patógeno. Una intervención que permite "hacer como si..." se abordaran ciertos problemas sin obligarnos a correr el riesgo de discutir el trabajo real, supone no correr el riesgo de movilizar las defensas y desestabilizar ese equilibrio que permite soportar la situación.

23 Esta investigación tiene como intención dejar en evidencia en qué medida la creación de espacios de deliberación es un eslabón intermedio indispensable para restablecer la relación entre trabajo y placer, cooperación y eventualmente ser fuente de una forma 
de emancipación. Para conseguirlo, un profundo trabajo de la demanda es indispensable, desde el punto de vista metodológico, aunque también y sobre todo ético y deontológico. Si un colectivo está dispuesto a iniciar una reflexión colectiva para lograr entender mejor su relación con el trabajo, se abre una vía de acción. Solo los colectivos de trabajo que esperan algo de ellos mismos, pueden de a poco hacerse cargo de los problemas que le son propios. Para ello, es necesario un trabajo previo de análisis de la demanda, de construcción de la investigación y una reflexión subsiguiente que apunte a elaborar una relación que pueda permitirles evolucionar en su pensamiento. En PDT se considera que, en la medida en que se evoluciona en el pensamiento, y sólo si se lo hace de manera colectiva, las vías de transformación saludables se abrirán, incluso para modificaciones de los aspectos materiales, del mobiliario, o para otros aspectos vinculados a la organización del trabajo.

Limitarnos a una racionalidad de las ciencias de los ingenieros, basada en la interface hombre máquina, o a una determinada ergonomía centrada en la productividad observable a través de cifras supone fracasar desde el inicio en la posibilidad de generar transformaciones en el trabajo que se encuentren al servicio de la salud. Si la deliberación colectiva y el trabajo de la demanda desempeñan un papel crucial es porque en PDT toda investigación es una acción que se orienta a la emancipación, y solo quienes realizan el trabajo pueden llevar adelante ese proceso. No puede prescribirse, aconsejarse ni indicarse. Por ello, tomar en consideración la racionalidad moral práctica de toda intervención es indispensable. Por lo antedicho, la psicopatología y la psicodinámica del trabajo no sólo aluden a la teoría de la actividad, sino también a la de la acción. El trabajo no puede ser tomado sólo por las categorías clásicas de la producción (poïesis). Implica, por una parte, categorías teóricas de la acción (praxis). Para entender la dimensión colectiva del trabajo tratando de interpretar la brecha entre coordinación y cooperación, se impone introducir la cuestión de la deliberación colectiva (Dejours, 2009). Esta actividad deóntica consiste en la construcción de normas de trabajo y de valores.

Como puede apreciarse, las diferencias entre las disciplinas de las que da cuenta esta tesis, son teóricas y metodológicas, pero también de orden político. Estas diferencias convocan a la subjetividad de los investigadores frente al campo que ellos abordan durante cada una de sus intervenciones. Se trata entonces de un debate entre disciplinas y, al mismo tiempo, de un debate entre investigadores, sean éstos ergónomos, clínicos del trabajo o expertos en evaluación de los RPS. Es el debate en torno de la posibilidad de construir un mundo del trabajo el que busca reforzar la posibilidad de emanciparnos.

No se trata de intentar transformar el trabajo simplemente por transformarlo. La mayoría de las transformaciones vividas en el mundo del trabajo a lo largo de las últimas décadas no han hecho más que agravar el estado de la salud mental de los trabajadores. Es por ello que resulta indispensable que las diferentes investigaciones se propongan como objetivo hacer oír la voz de aquellos que conocen mejor el trabajo: quienes lo hacen, quienes comprometen día a día su cuerpo (Dejours, 2001), su subjetividad, para cubrir la brecha entre el trabajo prescrito y el trabajo real. Como propone Dessors (2009), es indispensable poner el acento en el campo, en aquello que los trabajadores necesitan y en lo que esperan de nosotros como especialistas del trabajo, pero, sobre todo, en lo que ellos esperan de ellos mismos. Habrá que pensar aquello que, en cada contexto, en cada abordaje, permite hacer oír su voz, y facilita que 
puedan escucharse entre ellos, que son, en definitiva, los únicos que conocen el trabajo real y las dificultades frente a las que se ven confrontados diariamente, y por lo tanto, que conocen las vías posibles para su transformación. En la medida en que podamos hacer oír esas voces, podremos avanzar hacia una transformación del trabajo, de nuestras disciplinas y de nosotros mismos como investigadores, que constituirá un aporte, no a la alienación y a la soledad, sino a la emancipación, incluso la nuestra.

\section{BIBLIOGRAFÍA}

Askenazy, P., Baudelot, C., Brochard, P., Brun, J.P., Cases, C., Davezies, P., Falissard, B., Gallie, D., Gollac, M., Griffiths, A., Grignon, M., Imbernon, E., Leclerc, A., Molinier, P., Niedhammer, I., Parent-Thirion, A., Verger, D., Vézina, M., Volkoff, S., \& Weill-Fassina, A. (2011). Mesurer les facteurs psychosociaux de risque au travail pour les maitriser. Rapport du Collège d'expertise sur le suivi des risques psychosociaux au travail, faisant suite à la demande du Ministre du travail, de l'emploi et de la santé. París: http://travail-emploi.gouv.fr/IMG/pdf/

rapport_SRPST_definitif_rectifie_11_05_10.pdf.

Dejours, C. (1980/1990). Trabajo y desgaste mental. Una contribución a la psicopatología del trabajo. Buenos Aires: Área de Estudios e Investigaciones Laborales de la SECYT, CEIL/CONICET, CREDAL/ CNRS, Facultad de Psicología de la UBA, Hvmanitas.

Dejours, C. (1998/2006). La banalización de la injusticia social. Buenos Aires : Topía Editora.

Dejours, C. (2001). Le corps, d'abord - Corps biologique, corps érotique et sens moral. París: Payot.

Dejours, C. (2009). Travail vivant. Tomes I et II. París : Payot.

Dessors, D. (2009). De l'ergonomie à la psychodynamique du travail. Méthodologie de l'action. París : Érès éditions.

Ganem, V. (2012). La désobéissance à l'autorité. L'énigme de la Guadeloupe. París: PUF.

Karasek, R. A. (1979). Job demands, job decision latitude, and mental strain: Implications for job redesign. Administrative Science Quarterly, 24, 285-308. https://doi.org/10.2307/2392498

Karasek, R., Brisson, C., Kawakami, N., Houtman, I, Bongers, P., Amick, B. (1998). The Job Content Questionnaire (JCQ): An instrument for internationally comparative assessments of psychosocial job characteristics. Journal of Occupational Health Psychology, 3(4), 322-55. https://doi.org/ 10.1037/1076-8998.3.4.322

Lhuilier, D. (2005). Le " sale boulot ". Travailler, 2, 14, 3-98. https://doi.org/10.3917/trav.014.0073

Molinier, P. (2009). Des féministes et de leurs femmes de ménage : entre réciprocité du care et souhait de dépersonnalisation. Multitudes, 2, 37-38, 113-121. https://doi.org/10.3917/mult. 037.0113

Rolo, D. (2013). Contraintes organisationnelles, distorsion de la communication et souffrance éthique. Le cas des centres d'appels téléphoniques. Thèse de doctorat, Conservatoire national des arts et métiers, París. Rolo, D. (2014). Restricciones organizativas, distorsión de la comunicación y sufrimiento 
ético: el caso de los centros de llamadas. Laboreal, 10 (1), 79-83. http://dx.doi.org/10.15667/ laborealx0114dres

Siegrist, J., Starke, D., Chandola, T., Godin, I., Marmot, M., Niedhammer, I., \& Peter, R. (2004). The measurement of effort reward imbalance at work: European comparisons. Social Science \& Medicine, 58, 8, 1483-1499. https://doi.org/10.1016/S0277-9536(03)00351-4

Siegrist, J., Wege, N., Pühlhofer, F., \& Wahrendorf, M. (2008). A short generic measure of work stress in the era of globalisation: effort-reward imbalance. International archives of occupational and environmental health, 82, 1005-1013. https://doi.org/10.1007/s00420-008-0384-3

Sznelwar, L., Abrahão, J. I., \& Mascia, F. L. (2006). Trabalhar em centrais de atendimento: a busca de sentido em tarefas esvaziadas. Revista Brasileira de Saúde Ocupacional, 31, 114, 97-112. https:// doi.org/10.1590/S0303-76572006000200009

\title{
NOTAS
}

1. ODAM IX: $9^{\text {th }}$ International Symposium on Human Factors in Organizational Design and Management, Guarujá, Brasil, marzo 2008.

2. Suicide et travail : état de la recherche internationale. Travailler 2014/1 ( $\left.n^{\circ} 31\right) .200$ pages. ISSN: $1620-5340$

\author{
AUTOR \\ PATRICIO NUSSHOLD \\ Laboratoire PCPP (Psychologie Clinique, Psychopathologie, Psychanalyse) \\ Université Paris Descartes \\ Centre Henri Piéron, 71, av. Edouard Vaillant, 92774 Boulogne Billancourt Cedex France \\ patricio.nusshold@ast-i.org
}

